\title{
SOLUSI PERSAMAAN DIFERENSIAL ORDE DUA DENGAN SYARAT BATAS FRACTIONAL (KASUS AKAR KOMPLEKS)
}

\author{
DEBBY YOLANDA, BUDI RUDIANTO* \\ Program Studi S1 Matematika, \\ Fakultas Matematika dan Ilmu Pengetahuan Alam, Universitas Andalas, \\ Kampus UNAND Limau Manis Padang, Indonesia. \\ email : debyyolanda01@gmail.com,budirudianto@sci.unand.ac.id
}

Diterima 15 Desember 2020 Direvisi 29 Desember 2020 Dipublikasikan 12 Januari 2021

\begin{abstract}
Abstrak. Dalam artikel ini dikaji solusi persamaan diferensial biasa linier orde dua homogen dengan syarat batas berupa turunan fractional. Kajian ini khusus untuk persamaan karakteristik dari persamaan diferensial orde dua dengan akar bilangan kompleks murni. Turunan fractional yang digunakan dalam skripsi ini adalah turunan fractional Riemann-Liouville
\end{abstract}

Kata Kunci: Persamaan diferensial biasa linier orde dua homogen, Akar kompleks, Turunan Fractional Riemann-Liouville.

\section{Pendahuluan}

Persamaan diferensial adalah persamaan yang memuat turunan dari satu (atau beberapa) variabel tak bebas terhadap satu atau lebih variabel bebas. Persamaan diferensial biasa adalah persamaan yang memuat turunan biasa dari satu atau lebih variabel tak bebas terhadap satu variabel bebas [9].

Suatu persamaan diferensial biasa linier orde dua adalah suatu persamaan yang berbentuk sebagai berikut [3]:

$$
p(x) D^{2} y(x)+q(x) D y(x)+r(x) y(x)=g(x)
$$

dimana $D=\frac{d}{d x}$ dan $p(x), q(x), r(x)$ adalah fungsi-fungsi dari $x$ dengan $p(x) \neq$ 0. Jika $g(x)=0$, maka (1.1) disebut persamaan diferensial biasa linier homogen. Kajian tentang solusi persamaan diferensial (1.1) merupakan topik klasik dalam matematika. Eksistensi solusi dari persamaan (1.1) telah dijamin dalam [3]. Dalam makalah ini dikaji bagaimana bentuk solusi dari (1.1) pada suatu interval $(a, b)$, $a, b \in \mathbb{R}$ dengan syarat batas memuat turunan fractional Riemann-Liouville, dimana $p(x), q(x), r(x)$ adalah konstanta dengan $p(x) \neq 0$. Kajian ini merupakan elaborasi dari informasi dalam artikel [4].

*penulis korespondensi 


\section{Beberapa Konsep Dasar}

Definisi 2.1. [8] Fungsi Gamma yang dinotasikan dengan $\Gamma(n)$ didefinisikan sebagai berikut:

$$
\Gamma(n)=\int_{0}^{\infty} x^{n-1} e^{-x} d x, n>0 .
$$

Definisi 2.2. [6] Fungsi Beta didefinisikan sebagai berikut :

$$
B(p, q)=\int_{0}^{1} x^{p-1}(1-x)^{q-1} d x, x \in \mathbb{R} \text { dan } p, q \in \mathbb{R}, p>0, q>0 .
$$

Definisi 2.3. [1] Fungsi Mittag-Leffer dua parameter didefinisikan sebagai berikut:

$$
E_{\alpha, \beta}(z)=\sum_{k=0}^{\infty} \frac{z^{k}}{\Gamma(\alpha k+\beta)}, \quad \alpha>0, \beta>0, z \in \mathbb{C}
$$

Deret Taylor merupakan bentuk dari fungsi matematika sebagai jumlah tak hingga dari suku yang nilainya dihitung dari turunan fungsi tersebut pada suatu titik.

Teorema 2.4. [7] Misalkan $f$ adalah fungsi yang turunan $k e-(n+1)-n y a$ ada untuk setiap $x$ pada selang terbuka I yang mengandung a,maka untuk setiap di dalam I berlaku

$$
f(x)=f(a)+\frac{f^{\prime}(a)}{1 !}(x-a)+\frac{f^{(2)}(a)}{2 !}(x-a)^{2}+\cdots+\frac{f^{(n)}(a)}{n !}(x-a)^{n}+R_{n}(x)
$$

dengan sisa $R_{n}(x)$ dinyatakan oleh

$$
R_{n}(x)=\frac{f^{n+1}(\xi)}{(n+1) !}(x-a)^{n+1},
$$

dengan $\xi$ adalah titik antara $x$ dan a.

Persamaan diferensial orde dua adalah persamaan diferensial yang turunan tertingginya berorde dua. Secara umum persamaan diferensial biasa linier orde dua dapat ditulis dalam bentuk [3]:

$$
p(x) D^{2} y(x)+q(x) D y(x)+r(x) y(x)=g(x),
$$

dimana $p(x), q(x), r(x)$ adalah koefisien dari persaman diferensial tersebut.

Jika $g(x)=0$, maka persamaan (2.3) disebut persamaan diferensial biasa orde dua homogen. Selanjutnya dengan mengganti $p(x)=a_{2}, q(x)=a_{1}$, dan $r(x)=a_{0}$ maka persamaan (2.3) dapat ditulis kembali dalam bentuk [3]

$$
a_{2} D^{2} y(x)+a_{1} D y(x)+a_{0} y(x)=0 .
$$

Persamaan (2.4) dapat diselesaikan dengan menentukan terlebih dahulus akar-akar karakteristiknya, yaitu:

$$
r_{1}=\frac{-a_{1}+\sqrt{a_{1}^{2}-4 a_{2} a_{0}}}{2 a_{2}} \text { dan } r_{2}=\frac{-a_{1}-\sqrt{a_{1}^{2}-4 a_{2} a_{0}}}{2 a_{2}} .
$$

Adapun bentuk-bentuk penyelesaian persamaan karakteristik adalah sebagai berikut [3]. 
(1) Jika $a_{1}^{2}-4 a_{2} a_{0}>0$, maka akan diperoleh akar-akar riil berbeda $\left(r_{1} \neq r_{2}\right)$ dan solusi berupa:

$$
y(x)=C_{1} e^{r_{1} x}+C_{2} e^{r_{2} x}, \quad C_{1}, C_{2} \in \mathbb{R} .
$$

(2) Jika $a_{1}^{2}-4 a_{2} a_{0}=0$,maka akan diperoleh akar-akar riil sama $\left(r_{1}=r_{2}\right)$ dan solusi berupa:

$$
y(x)=C_{1} e^{r_{1} x}+x C_{2} e^{r_{2} x}, \quad C_{1}, C_{2} \in \mathbb{R} .
$$

(3) Jika $a_{1}^{2}-4 a_{2} a_{0}<0$, maka akan diperoleh akar-akar kompleks $\left(r_{1,2}=p \pm i q\right)$ dan solusi berupa:

$$
y(x)=e^{p x}\left(C_{1} \cos q x+C_{2} \sin q x\right), \quad C_{1}, C_{2} \in \mathbb{R} .
$$

Definisi 2.5. [1] Turunan fractional Riemann-Liouville kiri orde $\alpha$ dan kanan orde $\beta$ berturut-turut didefinisikan sebagai berikut:

$$
\begin{aligned}
& D_{a^{+}}^{\alpha} y(x)= \begin{cases}\frac{1}{\Gamma(n-\alpha)} \frac{d^{n}}{d x^{n}} \int_{a}^{x} \frac{y(t)}{(x-t)^{\alpha-n+1}} d t, & \text { untuk } \alpha \in \mathbb{R} \backslash \mathbb{N}_{0}, n=[\alpha]+1 \\
D^{n} y(x), & \text { untuk } \alpha=n \in \mathbb{N}_{0}\end{cases} \\
& D_{b^{-}}^{\beta} y(x)=\left\{\begin{array}{ll}
\frac{(-1)^{n}}{\Gamma(n-\beta)} \frac{d^{n}}{d x^{n}} \int_{x}^{b} \frac{y(t)}{(t-x)^{\beta-n+1}} d t, & \text { untuk } \beta \in \mathbb{R} \backslash \mathbb{N}_{0}, n=[\beta]+1 \\
(-1)^{n} D^{n} y(x), & \text { untuk } \beta=n \in \mathbb{N}_{0}
\end{array},\right.
\end{aligned}
$$

dimana $\mathbb{N}_{0}=\mathbb{N} \cup\{0\}$ dan $[\alpha]$ adalah bilangan bulat terbesar yang kurang dari atau sama dengan $\alpha,[\beta]$ adalah bilangan bulat terbesar yang kurang dari atau sama dengan $\beta$.

Berikut sifat-sifat untuk turunan fractional Riemann-Liouville kiri dan kanan:

(1) $D^{\alpha}(\lambda f(x))=\lambda D^{\alpha} f(x)$

(2) $D^{\alpha}\left(\lambda_{1} f(x)+\lambda_{2} g(x)\right)=D^{\alpha}\left(\lambda_{1} f(x)\right)+D^{\alpha}\left(\lambda_{2} g(x)\right)$.

Dengan mengggunakan Definisi 2.5 diperoleh turunan fractional dari fungsi pangkat sebagai berikut:

$$
\begin{aligned}
D_{a+}^{\alpha}(x-a)^{m} & =\frac{\Gamma(m+1)}{\Gamma(m+1-\alpha)}(x-a)^{m-\alpha}, \alpha \geq 0, m>-1, \\
D_{b-}^{\beta}(b-x)^{m} & =\frac{\Gamma(m+1)}{\Gamma(m+1-\beta)}(b-x)^{m-\beta}, \beta \geq 0, m>-1 .
\end{aligned}
$$

\section{Pembahasan}

Perhatikan persamaan diferensial berikut:

$$
D^{2} y(x)+k^{2} y(x)=0,
$$

dengan syarat batas berupa kombinasi dua dari persamaan berikut:

$$
\left.\mu_{1} D_{a^{+}}^{\alpha_{1}} y(x)\right|_{x=b}+\left.\mu_{2} D_{b-}^{\beta_{1}} y(x)\right|_{x=a}=L_{1}
$$




$$
\begin{aligned}
& \left.\mu_{3} D_{a^{+}}^{\alpha_{1}} y(x)\right|_{x=b}+\left.\mu_{4} D_{a+}^{\alpha_{2}} y(x)\right|_{x=b}=L_{2} \\
& \left.\mu_{5} D_{b-}^{\beta_{1}} y(x)\right|_{x=a}+\left.\mu_{6} D_{b-}^{\beta_{2}} y(x)\right|_{x=a}=L_{3}
\end{aligned}
$$

dimana $\alpha_{1}, \alpha_{2}, \beta_{1}, \beta_{2} \in[0,2], \alpha_{1} \neq \alpha_{2}, \beta_{1} \neq \beta_{2}, \mu_{i} \in \mathbb{R}, i=1,2, \cdots, 6$, dan $L_{j} \in$ $\mathbb{R}, j=1,2,3$, dengan $D_{a^{+}}^{\alpha_{m}}, D_{b^{-}}^{\beta_{n}}$, adalah turunan fractional Riemann-Liouville.

Solusi dari persamaan (3.1) adalah

$$
y(x)=C_{1} \cos (k x)+C_{2} \sin (k x) .
$$

dimana $C_{1}$ dan $C_{2}$ adalah konstanta sebarang.

Untuk mendapatkan solusi yang memenuhi syarat batas (3.2),(3.3), dan (3.4), perhatikan kasus-kasus berikut ini:

(1) Syarat batas berupa (3.2) dan (3.3)

Subtitusi solusi $y(x)$ kedalam syarat batas (3.2) dan (3.3), diperoleh:

$$
\begin{aligned}
& C_{1} A_{11}+C_{2} B_{11}=L_{1} \\
& C_{1} A_{21}+C_{2} B_{21}=L_{2}
\end{aligned}
$$

dimana

$$
\begin{aligned}
& A_{11}=\left.\mu_{1} D_{a+}^{\alpha_{1}} \cos (k x)\right|_{x=b}+\left.\mu_{2} D_{b-}^{\beta_{1}} \cos (k x)\right|_{x=a} \\
& B_{11}=\left.\mu_{1} D_{a+}^{\alpha_{1}} \sin (k x)\right|_{x=b}+\left.\mu_{2} D_{b-}^{\beta_{1}} \sin (k x)\right|_{x=a} \\
& A_{21}=\left.\mu_{3} D_{a+}^{\alpha_{1}} \cos (k x)\right|_{x=b}+\left.\mu_{4} D_{a+}^{\alpha_{2}} \cos (k x)\right|_{x=b} \\
& B_{21}=\left.\mu_{3} D_{a+}^{\alpha_{1}} \sin (k x)\right|_{x=b}+\left.\mu_{4} D_{a+}^{\alpha_{2}} \sin (k x)\right|_{x=b}
\end{aligned}
$$

(2) Syarat batas berupa (3.3) dan (3.4)

Subtitusi solusi $y(x)$ kedalam syarat batas (3.3) dan (3.4), diperoleh

$$
\begin{aligned}
& C_{3} A_{12}+C_{4} B_{12}=L_{2} \\
& C_{3} A_{22}+C_{4} B_{22}=L_{3}
\end{aligned}
$$

dimana

$$
\begin{aligned}
& A_{12}=\left.\mu_{3} D_{a+}^{\alpha_{1}} \cos (k x)\right|_{x=b}+\left.\mu_{4} D_{a+}^{\alpha_{2}} \cos (k x)\right|_{x=b} \\
& B_{12}=\left.\mu_{3} D_{a+}^{\alpha_{1}} \sin (k x)\right|_{x=b}+\left.\mu_{4} D_{a+}^{\alpha_{2}} \sin (k x)\right|_{x=b} \\
& A_{22}=\left.\mu_{5} D_{b-}^{\beta_{1}} \cos (k x)\right|_{x=a}+\left.\mu_{6} D_{b-}^{\beta_{2}} \cos (k x)\right|_{x=a} \\
& B_{22}=\left.\mu_{5} D_{b-}^{\beta_{1}} \sin (k x)\right|_{x=a}+\left.\mu_{6} D_{b-}^{\beta_{2}} \sin (k x)\right|_{x=a}
\end{aligned}
$$

(3) Syarat batas berupa (3.2) dan (3.4)

Subtitusi solusi $y(x)$ kedalam syarat batas (3.2) dan (3.4), diperoleh

$$
\begin{aligned}
& C_{5} A_{13}+C_{6} B_{13}=L_{1} \\
& C_{5} A_{23}+C_{6} B_{23}=L_{3}
\end{aligned}
$$


dimana

$$
\begin{aligned}
& A_{13}=\left.\mu_{1} D_{a+}^{\alpha_{1}} \cos (k x)\right|_{x=b}+\left.\mu_{2} D_{b-}^{\beta_{1}} \cos (k x)\right|_{x=a} \\
& B_{13}=\left.\mu_{1} D_{a+}^{\alpha_{1}} \sin (k x)\right|_{x=b}+\left.\mu_{2} D_{b-}^{\beta_{1}} \sin (k x)\right|_{x=a} \\
& A_{23}=\left.\mu_{5} D_{b-}^{\beta_{1}} \cos (k x)\right|_{x=a}+\left.\mu_{6} D_{b-}^{\beta_{2}} \cos (k x)\right|_{x=a} \\
& B_{23}=\left.\mu_{5} D_{b-}^{\beta_{1}} \sin (k x)\right|_{x=a}+\left.\mu_{6} D_{b-}^{\beta_{2}} \sin (k x)\right|_{x=a}
\end{aligned}
$$

Untuk menyelesaikan kasus-kasus diatas, perlu ditentukan terlebih dahulu turunan fractional Riemann-Liouville kiri dan kanan dari fungsi sinus dan cosinus dengan mengubah bentuk fungsi sinus dan cosinus menggunakan identitas trigonometri.

$$
\begin{aligned}
\left.D_{a+}^{\alpha} \sin (k x)\right|_{x=b} & =\left.D_{a+}^{\alpha} \sin (k(x-a)+k a)\right|_{x=b}, \\
& =\left.\cos (k a) D_{a+}^{\alpha} \sin (k(x-a))\right|_{x=b}+\left.\sin (k a) D_{a+}^{\alpha} \cos (k(x-a))\right|_{x=b}, \\
\left.D_{a+}^{\alpha} \cos (k x)\right|_{x=b} & =\left.D_{a+}^{\alpha} \cos (k(x-a)+k a)\right|_{x=b}, \\
& =\left.\cos (k a) D_{a+}^{\alpha} \cos (k(x-a))\right|_{x=b}-\left.\sin (k a) D_{a+}^{\alpha} \sin (k(x-a))\right|_{x=b} \\
\left.D_{b-}^{\beta} \sin (k x)\right|_{x=a} & =D_{b-}^{\beta}\left(-\left.\sin (k(b-x)-k b)\right|_{x=b}, \quad\right. \\
& =-\left.\cos (k b) D_{b-}^{\beta} \sin (k(b-x))\right|_{x=a}+\left.\sin (k b) D_{b-}^{\beta} \cos (k(b-x))\right|_{x=a}, \\
\left.D_{b-}^{\beta} \cos (k x)\right|_{x=a} & =\left.D_{b-}^{\beta} \cos (k(b-x)-k b)\right|_{x=a}, \\
& =\left.\cos (k b) D_{b-}^{\beta} \cos (k(b-x))\right|_{x=a}+\left.\sin (k b) D_{b-}^{\beta} \sin (k(b-x))\right|_{x=a} .
\end{aligned}
$$

Dengan menggunakan deret Taylor diperoleh

$$
\begin{aligned}
\left.D_{a+}^{\alpha} \sin (k(x-a))\right|_{x=b} & =\left.\sum_{s=0}^{\infty} \frac{(-1)^{s}(k)^{2 s+1}}{\Gamma(2 s+2)} D_{a+}^{\alpha}(x-a)^{2 s+1}\right|_{x=b}, \\
\left.D_{a+}^{\alpha} \cos (k(x-a))\right|_{x=b} & =\left.\sum_{s=0}^{\infty} \frac{(-1)^{s}(k)^{2 s}}{\Gamma(2 s+1)} D_{a+}^{\alpha}(x-a)^{2 s}\right|_{x=b} \\
\left.D_{b-}^{\beta} \sin (k(b-x))\right|_{x=a} & =\left.\sum_{s=0}^{\infty} \frac{(-1)^{s}(k)^{2 s+1}}{\Gamma(2 s+2)} D_{b-}^{\beta}(b-x)^{2 s+1}\right|_{x=a}, \\
\left.D_{b-}^{\beta} \cos (k(b-x))\right|_{x=a} & =\left.\sum_{s=0}^{\infty} \frac{(-1)^{s}(k)^{2 s}}{\Gamma(2 s+1)} D_{b-}^{\beta}(b-x)^{2 s}\right|_{x=a} .
\end{aligned}
$$

Turunan fractional diatas dapat diselesaikan dengan menggunakan definisi turunan fractional Riemann-Liouville untuk fungsi pangkat pada persamaan (2.8) 
sehingga diperoleh

$$
\begin{aligned}
\left.D_{a+}^{\alpha} \sin (k(x-a))\right|_{x=b} & =\left.k(x-a)^{1-\alpha} \sum_{s=0}^{\infty} \frac{(-1)^{s}(k(x-a))^{2 s}}{\Gamma(2 s+2-\alpha)}\right|_{x=b}, \\
\left.D_{a+}^{\alpha} \cos (k(x-a))\right|_{x=b} & =\left.(x-a)^{-\alpha} \sum_{s=0}^{\infty} \frac{(-1)^{s}(k)^{2 s}}{\Gamma(2 s+1-\alpha)}(x-a)^{2 s}\right|_{x=b}, \\
\left.D_{b-}^{\beta} \sin (k(b-x))\right|_{x=a} & =\left.k(b-x)^{1-\beta} \sum_{s=0}^{\infty} \frac{(-1)^{s}(k(b-x))^{2 s}}{\Gamma(2 s+2-\beta)}\right|_{x=a}, \\
\left.D_{b-}^{\beta} \cos (k(b-x))\right|_{x=a} & =\left.(b-x)^{-\beta} \sum_{s=0}^{\infty} \frac{(-1)^{s}(k(b-x))^{2 s}}{\Gamma(2 s+1-\beta)}\right|_{x=a} .
\end{aligned}
$$

Deret-deret yang membentuk persamaan di atas merupakan bentuk dari fungsi Mittag-Leffler, sehingga dapat ditulis

$$
\begin{aligned}
& D_{a+\left.\sin (k(x-a))\right|_{x=b} ^{\alpha}}^{\alpha}=k(b-a)^{1-\alpha} E_{2,2-\alpha}\left(-k^{2}(b-a)^{2}\right. \\
& \left.D_{a+}^{\alpha} \cos (k(x-a))\right|_{x=b}=(b-a)^{-\alpha} E_{2,1-\alpha}\left(-k^{2}(b-a)^{2}\right) \\
& \left.D_{b-}^{\beta} \sin (k(b-x))\right|_{x=a}=k(b-a)^{1-\beta} E_{2,2-\beta}\left(-k^{2}(b-a)^{2}\right) \\
& \left.D_{b-}^{\beta} \cos (k(b-x))\right|_{x=a}=(b-a)^{-\alpha} E_{2,1-\beta}\left(-k^{2}(b-a)^{2}\right)
\end{aligned}
$$

Subtitusi persamaan (3.8) ke turunan fractional Riemann-Lioville fungsi sinus dan cosinus yang diperoleh sebelumnya, sehingga diperoleh

$$
\begin{aligned}
\left.D_{a+}^{\alpha} \sin (k x)\right|_{x=b}= & k(b-a)^{1-\alpha} \cos (k a) E_{2,2-\alpha}\left(-k^{2}(b-a)^{2}\right)+ \\
& (b-a)^{-\alpha} \sin (k a) E_{2,1-\alpha}\left(-k^{2}(b-a)^{2}\right) \\
\left.D_{a+}^{\alpha} \cos (k x)\right|_{x=b}= & (b-a)^{-\alpha} \cos (k a) E_{2,1-\alpha}\left(-k^{2}(b-a)^{2}\right)- \\
& k(b-a)^{1-\alpha} \sin (k a) E_{2,2-\alpha}\left(-k^{2}(b-a)^{2}\right) \\
\left.D_{b-}^{\beta} \sin (k x)\right|_{x=a}= & -k(b-a)^{1-\beta} \cos (k b) E_{2,2-\beta}\left(-k^{2}(b-a)^{2}\right)+ \\
& (b-a)^{-\beta} \sin (k b) E_{2,1-\beta}\left(-k^{2}(b-a)^{2}\right) \\
\left.D_{b-}^{\beta} \cos (k x)\right|_{x=a}= & (b-a)^{-\beta} \cos (k b) E_{2,1-\beta}\left(-k^{2}(b-a)^{2}\right)+ \\
& k(b-a)^{1-\beta} \sin (k b) E_{2,2-\beta}\left(-k^{2}(b-a)^{2}\right)
\end{aligned}
$$

Contoh 3.1. Akan ditentukan solusi persamaan diferensial

$$
D^{2} y(x)+9 y(x)=0, x \in[2,4]
$$

dengan syarat batas

$$
\begin{aligned}
& \left.D_{2+}^{1 / 3} y(x)\right|_{x=4}+\left.D_{2+}^{1 / 4} y(x)\right|_{x=4}=1 \\
& \left.D_{2+}^{1 / 3} y(x)\right|_{x=4}+\left.D_{4-}^{1 / 4} y(x)\right|_{x=2}=2
\end{aligned} .
$$

Solusi dari (3.13) adalah

$$
y(x)=C_{1} \cos (3 x)+C_{2} \sin (3 x) .
$$


68 Debby Yolanda dan Budi Rudianto

Selanjutnya subtitusi $y(x)$ ke dalam syarat batas.

$$
\begin{aligned}
\left.D_{2+}^{1 / 3} y(x)\right|_{x=4}+\left.D_{2+}^{1 / 4} y(x)\right|_{x=4}= & \left.C_{1}\left[D_{2+}^{1 / 3} \cos (3 x)+D_{2+}^{1 / 4} \cos (3 x)\right]\right|_{x=4}+ \\
& \left.C_{2}\left[D_{2+}^{1 / 3} \sin (3 x)+D_{2+}^{1 / 4} \sin (3 x)\right]\right|_{x=4}
\end{aligned}
$$

Dengan menggunakan (3.9) dan (3.10) diperoleh

$$
\begin{aligned}
& {\left.\left[D_{2+}^{1 / 3} \cos (3 x)+D_{2+}^{1 / 4} \cos (3 x)\right]\right|_{x=4}=2,7321} \\
& {\left.\left[D_{2+}^{1 / 3} \sin (3 x)+D_{2+}^{1 / 4} \sin (3 x)\right]\right|_{x=4}=-0.3472}
\end{aligned}
$$

Sehingga,

$$
\begin{aligned}
& 2,7321 C_{1}-0,3472 C_{2}=1 \text {. } \\
& \left.D_{2+}^{1 / 3} y(x)\right|_{x=4}+\left.D_{4-}^{1 / 4} y(x)\right|_{x=2}=C_{1}\left[\left.D_{2+}^{1 / 3} \cos (3 x)\right|_{x=4}+\left.D_{4-}^{1 / 4} \cos (3 x)\right|_{x=2}\right]+ \\
& C_{2}\left[\left.D_{2+}^{1 / 3} \sin (3 x)\right|_{x=4}+\left.D_{4-}^{1 / 4} \sin (3 x)\right|_{x=2}\right] \text {. }
\end{aligned}
$$

Dengan menggunakan (3.9),(3.10),(3.11), dan (3.12) diperoleh

$$
\begin{aligned}
{\left[\left.D_{2+}^{1 / 3} \cos (3 x)\right|_{x=4}+\left.D_{4-}^{1 / 4} \cos (3 x)\right|_{x=2}\right] } & =2.6094 \\
{\left[\left.D_{2+}^{1 / 3} \sin (3 x)\right|_{x=4}+\left.D_{4-}^{1 / 4} \sin (3 x)\right|_{x=2}\right] } & =-0.8960 .
\end{aligned}
$$

Sehingga,

$$
2,6094 C_{1}-0,8960 C_{2}=2 .
$$

Selanjutnya akan ditentukan $C_{1}$ dan $C_{2}$ dari persamaan (3.15) dan (3.16).

$$
\left[\begin{array}{l}
2,6094-0,8960 \\
2,7321-0,3472
\end{array}\right]\left[\begin{array}{l}
C_{1} \\
C_{2}
\end{array}\right]=\left[\begin{array}{l}
2 \\
1
\end{array}\right] .
$$

Karena matriks $\left[\begin{array}{l}2,6094-0,8960 \\ 2,7321-0,3472\end{array}\right]$ nonsingular, maka

$$
\left[\begin{array}{l}
C_{1} \\
C_{2}
\end{array}\right]=\left[\begin{array}{l}
2,6094-0,8960 \\
2,7321-0,3472
\end{array}\right]^{-1}\left[\begin{array}{l}
2 \\
1
\end{array}\right]=\left[\begin{array}{c}
0,1307 \\
-1,8515
\end{array}\right] .
$$

Sehingga solusi dari (3.13) dengan syarat batas (3.14) adalah

$$
y(x)=0,1307 \cos (3 x)-1,8515 \sin (3 x) .
$$

\section{Kesimpulan}

Solusi persamaan diferensial

$$
D^{2} y(x)+k^{2} y(x)=0,
$$

dengan syarat batas berupa kombinasi dua dari persamaan berikut:

$$
\left.\mu_{1} D_{a^{+}}^{\alpha_{1}} y(x)\right|_{x=b}+\left.\mu_{2} D_{b-}^{\beta_{1}} y(x)\right|_{x=a}=L_{1}
$$




$$
\begin{aligned}
& \left.\mu_{3} D_{a^{+}}^{\alpha_{1}} y(x)\right|_{x=b}+\left.\mu_{4} D_{a+}^{\alpha_{2}} y(x)\right|_{x=b}=L_{2} \\
& \left.\mu_{5} D_{b-}^{\beta_{1}} y(x)\right|_{x=a}+\left.\mu_{6} D_{b-}^{\beta_{2}} y(x)\right|_{x=a}=L_{3}
\end{aligned}
$$

dimana $\alpha_{1}, \alpha_{2}, \beta_{1}, \beta_{2} \in[0,2], \alpha_{1} \neq \alpha_{2}, \beta_{1} \neq \beta_{2}, \mu_{i} \in \mathbb{R}, i=1,2, \cdots, 6$, dan $L_{j} \in \mathbb{R}, j=1,2,3$, dengan $D_{a^{+}}^{\alpha_{m}}, D_{b^{-}}^{\beta_{n}}$ adalah turunan fractional Riemann-Liouville dinyatakan sebagai berikut:

(1) Jika syarat batas berupa persamaan (3.2) dan (3.3), maka solusinya adalah

$$
y(x)=C_{1} \cos (k x)+C_{2} \sin (k x),
$$

dimana $C_{1}$ dan $C_{2}$ adalah solusi dari sistem persamaan linier berikut

$$
\begin{aligned}
& C_{1} A_{11}+C_{2} B_{11}=L_{1}, \\
& C_{1} A_{21}+C_{2} B_{21}=L_{2} .
\end{aligned}
$$

(2) Jika syarat batas berupa persamaan (3.3) dan (3.4), maka solusinya adalah

$$
y(x)=C_{3} \cos (k x)+C_{4} \sin (k x),
$$

dimana $C_{3}$ dan $C_{4}$ adalah solusi dari sistem persamaan linier berikut

$$
\begin{aligned}
& C_{3} A_{12}+C_{4} B_{12}=L_{2}, \\
& C_{3} A_{22}+C_{4} B_{22}=L_{3} .
\end{aligned}
$$

(3) Jika syarat batas berupa persamaan 3.2 dan 3.4, maka solusinya adalah

$$
y(x)=C_{5} \cos (k x)+C_{6} \sin (k x),
$$

dimana $C_{5}$ dan $C_{6}$ adalah solusi dari persamaan linier berikut

$$
\begin{aligned}
& C_{5} A_{13}+C_{6} B_{13}=L_{1}, \\
& C_{5} A_{23}+C_{6} B_{23}=L_{3},
\end{aligned}
$$

dimana $A_{11}, A_{21}, B_{11}, B_{21}, A_{12}, A_{22}, B_{12}, B_{22}, A_{13}, A_{23}, B_{13}, B_{23}$ dapat dilihat pada persamaan (3.5), (3.6), (3.7), dengan

$$
\begin{aligned}
\left.D_{a+}^{\alpha} \sin (k x)\right|_{x=b}= & k(b-a)^{1-\alpha} \cos (k a) E_{2,2-\alpha}\left(-k^{2}(b-a)^{2}\right)+ \\
& (b-a)^{-\alpha} \sin (k a) E_{2,1-\alpha}\left(-k^{2}(b-a)^{2}\right), \\
\left.D_{a+}^{\alpha} \cos (k x)\right|_{x=b}= & (b-a)^{-\alpha} \cos (k a) E_{2,1-\alpha}\left(-k^{2}(b-a)^{2}\right)- \\
& k(b-a)^{1-\alpha} \sin (k a) E_{2,2-\alpha}\left(-k^{2}(b-a)^{2}\right), \\
\left.D_{b-}^{\beta} \sin (k x)\right|_{x=a}= & -k(b-a)^{1-\beta} \cos (k b) E_{2,2-\beta}\left(-k^{2}(b-a)^{2}\right)+ \\
& (b-a)^{-\beta} \sin (k b) E_{2,1-\beta}\left(-k^{2}(b-a)^{2}\right), \\
\left.D_{b-}^{\beta} \cos (k x)\right|_{x=a}= & (b-a)^{-\beta} \cos (k b) E_{2,1-\beta}\left(-k^{2}(b-a)^{2}\right)+ \\
& k(b-a)^{1-\beta} \sin (k b) E_{2,2-\beta}\left(-k^{2}(b-a)^{2}\right) .
\end{aligned}
$$




\section{Ucapan Terima kasih}

Penulis mengucapkan terima kasih kepada Bapak Prof. Dr. Muhafzan, Ibu Riri Lestari, M.Si, Ibu Dr. Yanita, dan Ibu Dr. Arrival Rince Putri yang telah memberikan masukan dan saran dalam penyempurnaan penulisan artikel ini.

\section{Daftar Pustaka}

[1] A. A. Kilbas., H. M. Srivastava., J. J. Trujillo., 2006. Theory and Applications of Fractional Differential Equations. Elsevier: Amsterdam.

[2] Bartle. R.G dan Donald. R. Sherbert., 2010. Introduction to Real Analysis, Fourth Edition. USA: John Wiley and Sons.

[3] Boyce.W.E., Diprima.R.C., Elementary Differential Equation and Boundary Value Problems. Canada: John Wiley and Sons, Inc.

[4] Ciesielski. M., Blaszczyk.T., 2018. An Exact Solution of the Second-Order Differential Equation with the Fractional/Generalised Boundary Conditions. Vol. 2018, Article-ID 7283518: 1 - 9 .

[5] Diethelm.K. 2004. The Analysis of Fractional Differential Equation. Springer: New York

[6] Milici. C., Gheorghe. D., J. Tanreiro. 2019. Introduction to Fractional Dierential Equations. Springer: Switzerland.

[7] Purcell., Edwin. J., Dale Varberg., Steven. E. Rigdon., 2004. Kalkulus. Edisi Kedelapan. Jilid 2. Jakarta: Erlangga.

[8] Podlubny. I., 1999. Fractional Differential Equations. London: ACADEMIC PRES.

[9] Ross. S. R., 1996. Introduction To Ordinary Differential Equation, Third Edition. New York. 